\title{
Patenteamento em Nanotecnologia: Estudo do Setor de Materiais Poliméricos Nanoestruturados
}

\author{
Suzana Borschiver, Maria José O. C. Guimarães, Taís N. dos Santos, Flávio C. da Silva, Paulo Roberto C. Brum \\ Departamento de Processos Orgânicos, Escola de Química, UFRJ
}

\begin{abstract}
Resumo: Neste trabalho foi feito um estudo de tendências tecnológicas em nanotecnologia aplicado ao setor de materiais poliméricos, com base em informações extraídas de documentos de patentes. Foi usada como fonte de dados o banco de patentes da USPTO (United States Patent Trademark Office). Os dados foram obtidos via web, utilizando-se diversas palavras-chaves Foram mapeados os principais países depositantes, tipo de depositante e ano de aplicação, setores de aplicação, tipos de polímeros utilizados e principais aditivos e cargas incorporados às matrizes poliméricas.
\end{abstract}

Palavras-chave: Polímeros nanoestruturados, patenteamento, nanotecnologia, nanopolímeros.

\section{Patenting in Nanotechnology: Study of Nanostructurated Polymeric Materials Sector}

Abstract: In this work a study of technological tendencies in nanotechnology applied to polymeric materials sector was carried out, based on information extracted of paten documents. The patent office of USPTO (United States Patent Trademark Office) was used as a data source. The data were supplied via web, using several keywords. A mapping was made of the major countries contributing, types and year of patent deposition, application sectors, polymer types used, main additives and fillers incorporated to the polymeric matrices.

Keywords: Nanostructurated polymers, patentment, nanotechnology, nanopolymer.

\section{Introdução}

O termo Nanotecnologia vem revolucionando o mundo da ciência, trazendo muita expectativa a este setor que tem como principal característica sua diversificação. O objetivo da nanotecnologia é criar novos materiais e desenvolver novos produtos e processos baseados na crescente capacidade da tecnologia moderna de manipular átomos e moléculas. As áreas de aplicação da nanotecnologia abrangem praticamente todos os setores industriais e de serviços. A multiplicidade de aplicações é imensa e demanda conhecimentos multidisciplinares, baseados na física, química, biologia, ciência e engenharia de materiais, computação, entre outras áreas, que visam estender a capacidade humana de manipular a matéria até os limites do átomo ${ }^{[1,2]}$.

Atualmente, já existem iniciativas de vários países em investir em nanotecnologia. A maioria dos países desenvolvidos possui um setor bem estruturado nesta área, com programas científicos que impulsionam o governo a liberar verbas para pesquisa, incentivando as empresas a utilizarem esta tecnologia de forma a melhorar o desempenho de seus produtos ${ }^{[3]}$.

Muitas empresas por todo o mundo têm investido em nanotecnologia porém, é nos Estados Unidos onde se encontram a maioria destas. Na área de materiais, o potencial da Nanotecnologia é imenso. Novos materiais poliméricos nanoestruturados estão sendo desenvolvidos, com propriedades superiores aos já existentes. Além disso, a própria forma de produção desses materiais tenderá a sofrer transformações profundas. Argilas como a montmorilonita, da qual a Brasil possui extensa jazida, podem ser usadas para a produção de nanocompósitos. Em termos econômicos, o impacto dessa iniciativa será expressivo, pois as indústrias de materiais poliméricos movimentam bilhões de dólares por ano e representam um dos setores mais importantes da economia global. Vários países, incluindo o Brasil, apresentam reservas importantes de argilas de qualidade, que poderiam ser exploradas para aplicações nessa área. ${ }^{[4-9]}$.

Este trabalho teve como objetivo realizar um monitoramento informacional em nanotecnologia, com ênfase no setor de materiais poliméricos, com base em informações extraídas de documentos de patentes.

\section{Metodologia}

Foi utilizada como fonte de dados o banco de patentes da USPTO (United States Patent and Trademark Office) ${ }^{[10]}$, selecionando a opção das palavras - chave que constassem tanto do resumo como do título das patentes. O estudo de prospecção tecnológica foi feito com um volume de patentes publicadas entre 1976 e junho de 2004. Ao final da pesquisa, foram registradas um total de 852 patentes e com

Autor para correspondência: Suzana Borschiver, Escola de Química, UFRJ, Centro de Tecnologia, Bloco E, Sala E-204, Cidade Universitária, CEP: 21949900, Rio de Janeiro, RJ. E-mail: suzana@eq.ufr..br 


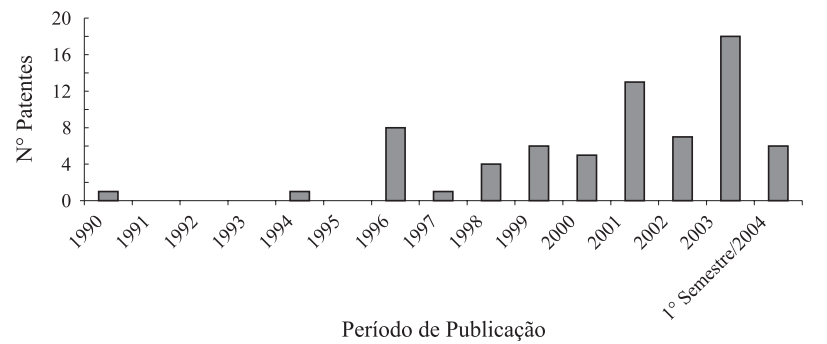

Figura 1. Evolução do registro de patentes em materiais poliméricos no período de 1990-2004

exclusão das repetições, o universo reduziu-se para 542. Dessas 542 patentes que apresentaram atuação em nanotecnologia, 70 eram relacionadas à materiais poliméricos nanoestruturados. Estas foram analisadas mais detalhadamente, para mapeamento dos principais países depositantes, evolução (ano de aplicação), tipos de depositantes e os setores de aplicação.

\section{Resultados e Discussão}

A Figura 1 apresenta a evolução do registro de patentes em materiais poliméricos. A primeira patente relacionada a um material polimérico nanoestruturado foi registrada em 1990 e a segunda somente em 1994. Houve um grande crescimento a partir de 1996, ressaltando-se também os anos de 2001 e 2003

Quanto à origem dessas patentes foram mapeados os principais países depositantes e os tipos de depositantes tais como Empresas, Centros de Pesquisa, Universidades e Pessoas Físicas.

\section{Distribuição por Países Depositantes}

A Figura 2 apresenta um panorama geral de todos os países depositantes de patentes nos últimos 28 anos. A distribuição por países depositantes apontou os Estados Unidos como o principal responsável pela maioria das patentes envolvendo materiais poliméricos nanoestruturados, respondendo por mais de 55\% do total destas, seguido pela França com aproximadamente $13 \%$ das patentes registradas.

\section{Distribuição por Tipos de Depositantes}

A Figura 3 mostra a grande participação das empresas (mais de 58\% de atuação); seguida das universidades, cen-

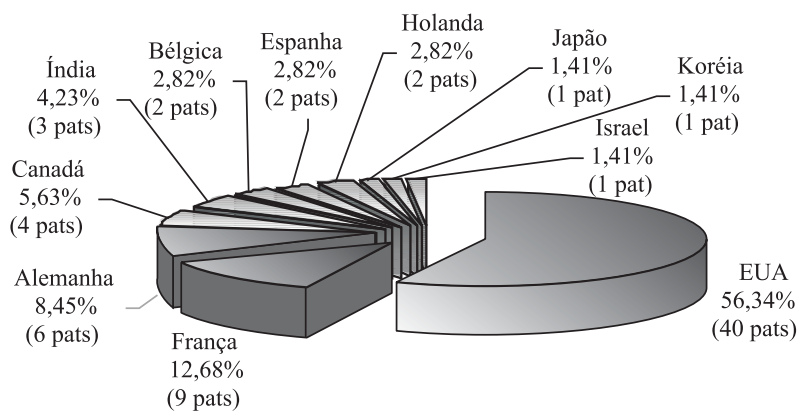

Figura 2. Países depositantes de patentes em materiais poliméricos nanoestruturados

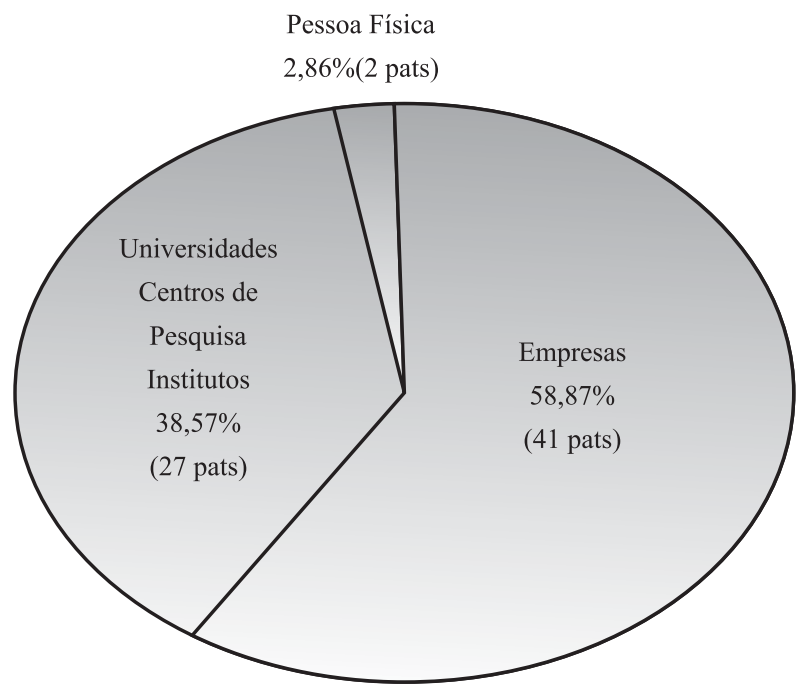

Figura 3. Tipos de depositantes

tros de pesquisas e institutos (um pouco mais de 38\%); e pessoas físicas (com uma parcela de aproximadamente 3\%) respectivamente. As principais empresas identificadas foram a NanoSystem L.L.C (5 patentes), empresa do setor farmacêutico, a Rhone-Poulenc Rorer S.A ( 3 patentes) e a L'Oreal ( 3 patentes), empresas do setor de fármacos e cosméticos respectivamente.

Foram identificados cerca de 103 órgãos diferentes, dentre os quais estão as universidades, centros de pesquisas e institutos, com registros de patentes. Na Tabela 1 estão compilados os órgãos com maior número de registros de patentes em nanomateriais.

Tabela 1. Principais Órgãos com Registros de Patentes

\begin{tabular}{ccc}
\hline Órgãos & País & $\mathbf{N}^{\mathbf{0}}$ de Registro \\
\hline $\begin{array}{c}\text { Southwest Research } \\
\text { Institute }\end{array}$ & Estados Unidos & 4 \\
$\begin{array}{c}\text { Massachusetts Institute of } \\
\text { Technology }\end{array}$ & Estados Unidos & 3 \\
$\begin{array}{c}\text { Board of Reagents of the } \\
\text { University of Texas System } \\
\text { Brown University } \\
\text { Foundation }\end{array}$ & Estados Unidos & 3 \\
$\begin{array}{c}\text { The Penn Stats Research } \\
\text { Foundation }\end{array}$ & Estados Unidos & 3 \\
$\begin{array}{c}\text { The United States of } \\
\text { America as represented by } \\
\text { the Secretary of the Navy } \\
\text { Institute fur }\end{array}$ & Estados Unidos & 2 \\
$\begin{array}{c}\text { Diagnostikforschung GmbH } \\
\text { Der Freien Universitat } \\
\text { Berline }\end{array}$ & Alemanha & 2 \\
$\begin{array}{c}\text { Dabur Research Foundation } \\
\text { Universidade de Santiago de } \\
\text { Compostela }\end{array}$ & Alemanha & 2 \\
\hline Índia & 2 \\
\hline
\end{tabular}




\section{Distribuição por Aplicações}

Os setores de aplicações dos materiais poliméricos nanoestruturados encontram-se descritos na Tabela $2 \mathrm{em}$ ordem decrescente de atuação. Esses setores são definidos separadamente, para que se entenda melhor cada um deles.

Suportes: São polímeros que possuem a função de suportar os materiais nanoestruturados em sua matriz. Um exemplo são os metais condutores que são inseridos em matrizes poliméricas com a finalidade de repassar esta propriedade ao polímero.

Processos de Fabricação de polímeros: Esta aplicação trata especificamente da síntese de um material polimérico nanoestruturado. Muitas vezes o material polimérico nanoestruturado é obtido pela redução do tamanho do polímero, tornando suas dimensões nanométricas, ou por meio de polimerização em microemulsão, formando assim o polímero dentro das micelas, o que garante a formação de partículas nanométricas.

Diagnósticos e Terapias: Os materiais poliméricos nanoestruturados podem ser aplicados em diagnósticos e terapias, como por exemplo, na desativação de moléculas causadoras de doenças como o câncer. Nestes processos as células causadoras da doença, são especificamente envolvidas por uma camada de material polimérico que causa desativação das mesmas.

Fármacos: Utilização de nanopartículas poliméricas na fabricação de medicamentos e cosméticos. Neste caso, os polímeros atuam na formulação dos produtos.

Medicina Reparadoura e Odontologia: Aplicação de materiais poliméricos nanoestruturados em medicina estética principalmente empregados no tratamento de pele, processo de colagem de ossos ou cartilagens.

Filmes Finos para Tratamento de Superfícies: São camadas incorporadas às superfícies com o intuito de proporcionar novas propriedades, tais como maior resistência, regularidade, anticorrosividade, entre outras. A maioria dos filmes utilizados para tratamento de superfícies possui silício incorporado à estrutura do polímero.

Tabela 2. Setores de Aplicação dos Materiais Poliméricos

\begin{tabular}{cc}
\hline Setor de Aplicação & $\mathbf{N}^{\mathbf{0}}$ de Registros \\
\hline Suportes Poliméricos & 21 \\
Processos de Fabricação de polímeros & 15 \\
Diagnósticos e Terapias & 10 \\
Fármacos & 9 \\
Medicina Reparadora e Odontologia & 8 \\
$\begin{array}{c}\text { Processo de Caracterização de } \\
\text { Partículas Específicas }\end{array}$ & 7 \\
Filmes Finos para Tratamento de \\
Superfícies
\end{tabular}

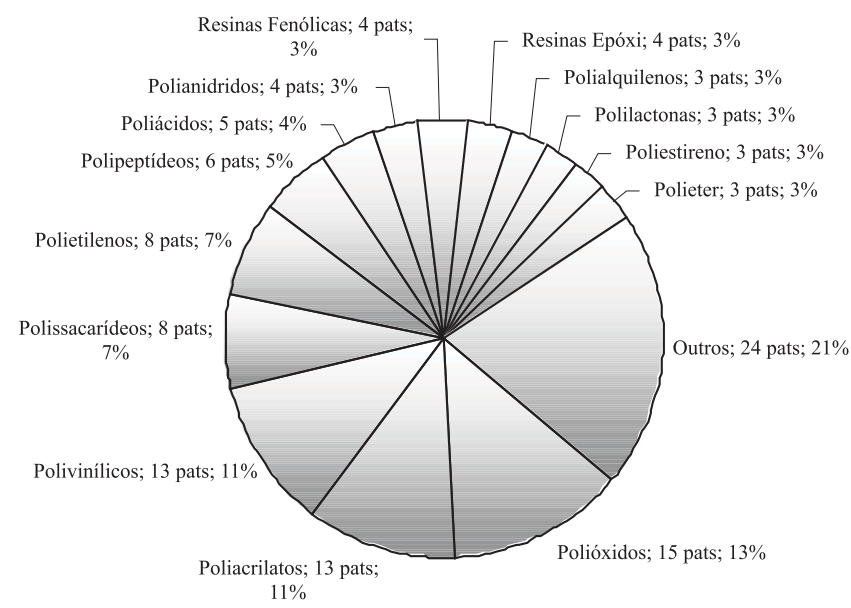

Figura 4. Polímeros utilizados em Nano Materiais

\section{Especificação dos Materiais Poliméricos Nanoestruturados}

\section{Tipos de Polímeros}

A Figura 4 apresenta um panorama dos principais tipos de polímeros identificados com seus respectivos percentuais de participação e número de registros. Pode-se observar que os polímeros mais utilizados no universo estudado são os polióxidos, poliacrilatos e os polivinílicos, que contribuem com mais de $35 \%$ dos registros envolvendo materiais poliméricos nanoestruturados.

Dentre os polióxidos destacam-se os polióxidos de etileno, propileno, etileno-propileno, entre outros. $\mathrm{Na}$ classe dos poliacrilatos encontram-se os poli (acrilatos de alquila), polimetacrilato, o poli(ácido acrílico), enquanto que no grupo dos polivinílicos destaca-se o poli(álcool vinílico) e derivados.

$\mathrm{Na}$ Figura 5 são apresentados os tipos de polímeros nanoestruturados. Pode-se verificar que quase a metade das patentes envolvendo materiais poliméricos nanoestruturados trata-se de homopolímeros, seguido dos copolímeros, das blendas e dos biopolímeros.

\section{Materiais incorporados aos polímeros}

Em muitos casos, à matriz polimérica são adiconados outros materiais que atuam como aditivos e/ou cargas. Esses

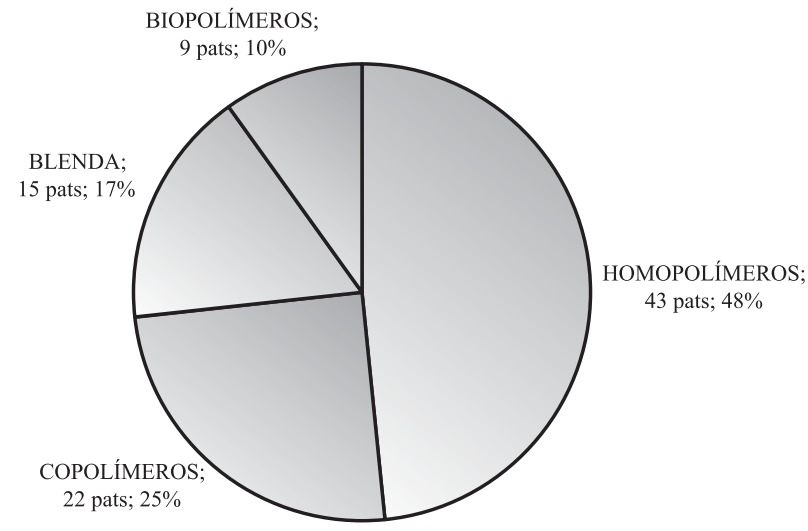

Figura 5. Tipos de Materiais Poliméricos 


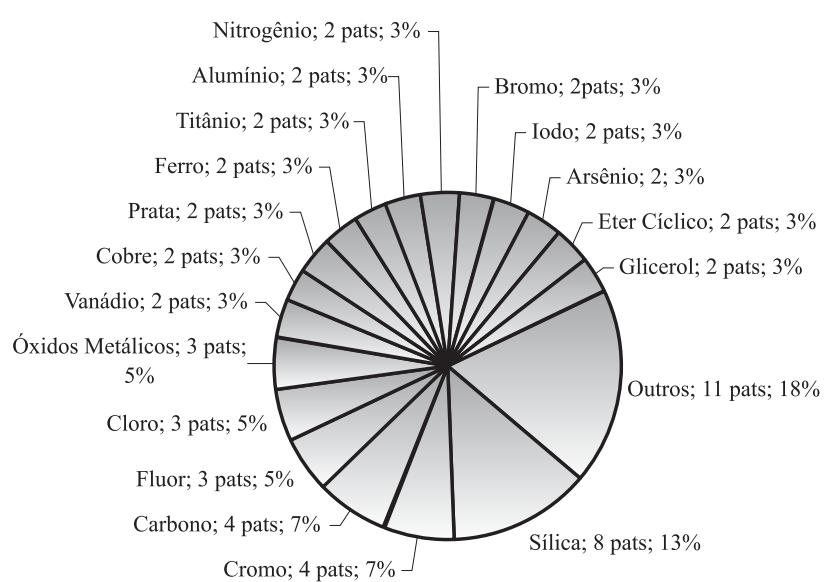

Figura 6. Materiais Incorporados aos polímeros

materiais, que se encontram em escala nanométrica, têm como objetivo fornecer uma característica específica, agindo sempre de forma a promover melhorias nas propriedades do material. A Figura 6 apresenta os diferentes materiais que podem ser incorporados aos polímeros, considerando seus percentuais de participação e respectivos números de registros de patentes. Pode-se observar a predominância do silício e seus derivados na composição dos materiais poliméricos.

\section{Conclusões}

A partir do estudo realizado no período de 1976 a junho de 2004, com análise de 542 patentes de Nanotecnologia e 70 de materiais poliméricos nanoestruturados pode-se obter algumas conclusões, tais como:

As primeiras patentes envolvendo Nanotecnologia começaram a ser publicadas na metade da década de 80 , havendo a partir de então um crescente aumento no número de depósitos. Patentes envolvendo materiais poliméricos nanoestruturados, só apareceram na década de 90.

Observa-se que mais de $70 \%$ das patentes sobre materiais poliméricos nanoestruturados foram depositadas pelos Estados Unidos, França e Alemanha. Quanto aos tipos de depositante, as empresas foram as que tiveram o maior número de depósitos, seguida das universidades, centros de pesquisa, institutos e das pessoas físicas. A Nanosystem S.A, empresa americana especializada na produção de fármacos, foi a que apresentou maior número de depósitos de patentes em mate- riais poliméricos nanoestruturados. Quanto às aplicações, nota-se que os maiores setores são os de suportes poliméricos e processos de fabricação de polímeros, atuando com mais de $50 \%$ do total. Em relação aos polímeros, observa-se que os mais utilizados em materiais nanoestruturados são os polióxidos, poliacrilatos, polivinílicos, polissacarídeos e polietilenos, correspondendo a um percentual de aproximadamente $50 \%$ das patentes analisadas. Quanto à questão dos principais materiais incorporados nas matrizes poliméricas, destaca-se o silício seguido do cromo e do carbono. Finalmente, pôde-se constatar o grande potencial dos polímeros nanoestruturados em diversas áreas do conhecimento da nanotecnologia.

\section{Referências Bibliográficas}

1. Santos, T. N.; Silva, F. C.; Brum, P. R. C.; Borschiver, S. \& Guimarães, M. J. O. C. - " Gestão do Conhecimento em Nanotecnologia e Nanociência com ênfase em Materiais Poliméricos Nanoestruturados", Publicação Interna, EQ/UFRJ (2004).

2. www.comciencia.br/nanotecnologia, agosto (2004).

3. Silva, C. G. - "Uma Introdução a Nanotecnologia", Cadernos de Estudos Avançados, Rio de Janeiro (2003).

4. Wongtschowski, P. - "Indústria Química: Riscos e Oportunidades", Editora Edgard Blücher Ltda $2^{\mathrm{a}}$ ed. (2002).

5. Chung, S. C.; Hahm, W. G.; Im, S. S. \& Oh, S.G. - Macrom. Res. 10, 221 (2002).

6. Fu, X. \& Qutubuddin, S. - Polymer, 10, 807 (2001).

7. Zeng, Q.H.; Wang, D.Z.; Yu, A.B.\& Lu, G.Q. Nanotechnology, 13, 459 (2002).

8. Esteves. A. C. C.; Timmons, A. B. \& Trindade, T. - Química Nova, 27 (5), 20 (2004).

9. Liu, T.; Burger, C. \& Chu, B. - Prog. Polym. Sci., 28, 5 (2003).

10. www.uspto.gov, junho (2004).

Enviado: 07/03/05

Reenviado: $18 / 07 / 05$

Aprovado: 25/07/05 